\title{
Long-term outcome after pulmonary endarterectomy for chronic thromboembolic pulmonary hypertension
}

\author{
Keiichi Ishida, $\mathrm{MD}, \mathrm{PhD},{ }^{\mathrm{a}}$ Masahisa Masuda, $\mathrm{MD}, \mathrm{PhD},{ }^{\mathrm{c}}$ Nobuhiro Tanabe, $\mathrm{MD}, \mathrm{PhD},{ }^{\mathrm{b}}$ \\ Goro Matsumiya, $\mathrm{MD}, \mathrm{PhD},{ }^{\mathrm{a}}$ Koichiro Tatsumi, $\mathrm{MD}, \mathrm{PhD},{ }^{\mathrm{b}}$ and Nobuyuki Nakajima, $\mathrm{MD}, \mathrm{PhD}{ }^{\mathrm{a}}$
}

\begin{abstract}
Objectives: Pulmonary endarterectomy is the treatment of choice for chronic thromboembolic pulmonary hypertension. Although several reports demonstrated excellent medium-term survival after pulmonary endarterectomy, long-term outcomes remain unclear. We reviewed long-term outcomes and determined risk factors for early and late adverse events.
\end{abstract}

\begin{abstract}
Methods: Seventy-seven patients were studied. Mean pulmonary arterial pressure was $47 \pm 10 \mathrm{~mm} \mathrm{Hg}$ and pulmonary vascular resistance was $868 \pm 319 \mathrm{dyne} \cdot \mathrm{s} \cdot \mathrm{cm}^{-5}$. Disease was classified as chronic thromboembolic pulmonary hypertension type $1(n=61)$, type $2(n=12)$, or type $3(n=4)$. Median and maximum follow-up periods were 5.6 and 20 years, respectively.

Results: There were 11 in-hospital deaths. Nonsurvivors had significantly higher mean pulmonary arterial pressure and pulmonary vascular resistance than did survivors $(54 \pm 10$ vs $46 \pm 10 \mathrm{~mm} \mathrm{Hg} ; P=.02 ; 1124 \pm 303$ vs $824 \pm 303$ dyne $\left.\cdot \mathrm{s} \cdot \mathrm{cm}^{-5} ; P<.01\right)$. In multivariate analysis, preoperative pulmonary vascular resistance was associated with in-hospital death (odds ratio, $1.003 ; 95 \%$ confidence interval, $1.001-1.005 ; P<.01$ ). During follow-up, there were 10 all-cause deaths, including 5 related to chronic thromboembolic pulmonary hypertension. Freedom from adverse events, including disease-specific death or New York Heart Association functional class III, was $70 \%$ at 10 years. In the Cox proportional hazard model, postoperative mean pulmonary arterial pressure was associated with adverse events (hazard ratio, 1.12; 95\% confidence interval, 1.03-1.21; $P<.01)$. Receiver operating characteristic curve analysis showed mean pulmonary arterial pressure of 34 $\mathrm{mm} \mathrm{Hg}$ as cutoff for adverse events.
\end{abstract}

Conclusions: Pulmonary endarterectomy had sustained favorable effects on long-term survival. High pulmonary vascular resistance was associated with in-hospital death, and postoperative mean pulmonary arterial pressure was an independent predictor of adverse events. (J Thorac Cardiovasc Surg 2012;144:321-6)

Chronic thromboembolic pulmonary hypertension $(\mathrm{CTEPH})$ is a life-threatening disease caused by unresolved, organized thrombi obstructing the pulmonary arteries. ${ }^{1,2}$ Progressive pulmonary hypertension severely compromises both clinical functional status and exercise capacity as a result of ventilation-perfusion mismatch and decreased cardiac output. In patients who do not undergo operation, the prognosis is disappointing and is determined by the severity of the pulmonary hypertension: if mean pulmonary arterial pressure (mPAP) exceeds 30 $\mathrm{mm} \mathrm{Hg}$, the 5-year survival is less than $30 \%$, and if it exceeds $50 \mathrm{~mm} \mathrm{Hg}$, the 5 -year survival is as low as $10 \%{ }^{3}$

\footnotetext{
From the Departments of Cardiovascular Surgery ${ }^{\mathrm{a}}$ and Respirology, ${ }^{\mathrm{b}}$ Graduate School of Medicine, Chiba University, Chiba, Japan; and the Department of Cardiovascular Surgery, ${ }^{\mathrm{c}}$ National Hospital Organization, Chiba Medical Center, Chiba, Japan.

Disclosures: Authors have nothing to disclose with regard to commercial support.

Received for publication May 28, 2011; revisions received Aug 9, 2011; accepted for publication Sept 15, 2011; available ahead of print Oct 12, 2011.

Address for reprints: Keiichi Ishida, MD, PhD, Department of Cardiovascular Surgery, Graduate School of Medicine, Chiba University, 1-8-1 Inohana Chuo-ku Chiba, 260-8670, Japan (E-mail: keiichi-ishida@pro.odn.ne.jp). $0022-5223 / \$ 36.00$

Copyright (c) 2012 by The American Association for Thoracic Surgery doi:10.1016/j.jtcvs.2011.09.004
}

Pulmonary endarterectomy (PEA), which has been established as a standard surgical treatment for CTEPH, is performed worldwide, mostly at centers with experience. ${ }^{1,4}$ PEA offers immediate and substantial decreases in both mPAP and pulmonary vascular resistance (PVR) and an increase in the cardiac index. ${ }^{4,5}$ Medium-term follow-up results for PEA reveal favorable effects on survival and clinical functional status ${ }^{6-10}$; however, the long-term outcomes remain unclear because of the limited available data. We began our PEA program in 1986 and have thus been performing the operation now for more than 20 years. Early on in our series, we performed PEA through a lateral thoracotomy; however, we have performed PEA through median sternotomy with intermittent deep hypothermic circulatory arrest according to the San Diego group procedure since $1990 .^{4,11,12}$ Here we review the long-term follow-up outcomes of a consecutive series of patients who underwent PEA through median sternotomy, and we seek to determine the factors influencing both early and late survival and functional status.

\section{MATERIALS AND METHODS}

Between 1990 and 2010, a total of 77 patients underwent PEA at Chiba University Hospital and affiliated hospitals. We retrospectively reviewed 


\section{Abbreviations and Acronyms \\ $\mathrm{CTEPH}=$ chronic thromboembolic pulmonary hypertension \\ mPAP $=$ mean pulmonary arterial pressure \\ NYHA $=$ New York Heart Association \\ PEA = pulmonary endarterectomy \\ PVR = pulmonary vascular resistance}

test and for categoric variables with the $\chi^{2}$ test or the Fisher's Exact test. We calculated the incidence rates for all-cause mortality, disease-specific mortality, and adverse events, including disease-specific mortality and impaired functional status in NYHA functional class III, and we used the Kaplan-Meier method to estimate survivals. Univariate and multivariate stepwise analyses with the Cox proportional hazard model were performed to identify the risk factors for disease-specific death and adverse events. The optimal PVR cutoff point for in-hospital death and the optimal mPAP cutoff value for adverse events were determined with the aid of a receiver operating characteristic curve. Results are expressed as hazard ratios with $95 \%$ confidence intervals.

\section{RESULTS}

\section{Early Results}

There were 11 in-hospital deaths among the cohort studied $(14 \%)$. The causes of death were as follows: right heart failure $(\mathrm{n}=6)$, pulmonary hemorrhage $(\mathrm{n}=2)$, cardiac tamponade $(\mathrm{n}=2)$, and reperfusion lung edema $(\mathrm{n}=1)$. With the exception of the 2 patients who died of cardiac tamponade, the other 9 patients had persistent pulmonary hypertension develop, and there were 5 patients who could not be weaned from cardiopulmonary bypass. Among the 66 PEA survivors, mPAP and PVR decreased (from $47 \pm$ $10 \mathrm{~mm} \mathrm{Hg}$ to $25 \pm 10 \mathrm{~mm} \mathrm{Hg}, P<.0001$; from $868 \pm$ 319 dyne $\cdot \mathrm{s} \cdot \mathrm{cm}^{-5}$ to $313 \pm 206$ dyne $\cdot \mathrm{s} \cdot \mathrm{cm}^{-5}$, $P<.0001$; respectively), and cardiac index increased (from $2.5 \pm 0.7 \mathrm{~L} /\left[\mathrm{min} \cdot \mathrm{m}^{2}\right]$ to $3.1 \pm 0.6 \mathrm{~L} /\left[\mathrm{min} \cdot \mathrm{m}^{2}\right]$; $P<.0001)$. Comparisons between survivors and nonsurvivors in terms of preoperative data are shown in Table 2. In the multivariate analysis, only preoperative PVR (odds ratio, 1.003; 95\% confidence interval, 1.001-1.005; $P<.01)$ was an independent predictor of in-hospital death. To determine the cutoff point for the influence of preoperative PVR on in-hospital death, we performed receiver operating characteristic curve analysis. This revealed a preoperative PVR of 1052 dyne $\cdot \mathrm{s} \cdot \mathrm{cm}^{-5}$ as the cutoff value for in-hospital death (area under the curve, 0.76; sensitivity, 0.64; specificity, 0.83).

\section{Late Results}

During the follow-up period, there were 10 all-cause deaths. Four deaths of right heart failure and 1 sudden cardiac death were regarded as being related to CTEPH. These patients exhibited NYHA functional class III symptoms as a result of persistence or worsening of pulmonary hypertension: 3 patients had persistent pulmonary hypertension with less than $10 \%$ decrease in PVR, and 2 patients had worsening of pulmonary hypertension despite early postoperative decrease in PVR (from 618 dyne $\cdot \mathrm{s} \cdot \mathrm{cm}^{-5}$ to 386 dyne $\mathrm{s} \cdot \mathrm{cm}^{-5}$ and from 1288 dyne $\cdot \mathrm{s} \cdot \mathrm{cm}^{-5}$ to 507 dyne $\cdot \mathrm{s} \cdot$ $\mathrm{cm}^{-5}$ ). Other causes of death included suffocation hematemesis, interstitial pneumonia, brain hemorrhage, and stroke. Among the operative survivors, clinical functional status improved markedly relative to the preoperative status. At the most recent follow-up at a mean of 6.5 years, 56 patients 
TABLE 1. Baseline patient characteristics

\begin{tabular}{lc}
\hline \multicolumn{1}{c}{ Variable } & Preoperative \\
\hline Male (no.) & $34(44 \%)$ \\
Age (y, mean \pm SD) & $55 \pm 11$ \\
Coagulation abnormality (no.) & $24(31 \%)$ \\
Deep vein thrombosis (no.) & $37(48 \%)$ \\
Insertion of inferior vena cava filter (no.) & $65(84 \%)$ \\
Duration of illness (mo, mean \pm SD) & $49.6 \pm 40.4$ \\
New York Heart Association functional class (no.) & \\
I & 0 \\
II & $16(21 \%)$ \\
III & $54(70 \%)$ \\
IV & $7(9 \%)$ \\
\hline
\end{tabular}

$(92 \%)$ were in NYHA functional class I or II, and 35 patients $(63 \%)$ had been weaned from home oxygen therapy. Among 5 patients in NYHA functional class III, 4 patients had worsening of pulmonary hypertension at follow-up. Their PVR decreased from $795 \pm 245$ dyne $\cdot \mathrm{s} \cdot \mathrm{cm}^{-5}$ to $398 \pm 146$ dyne $\cdot \mathrm{s} \cdot \mathrm{cm}^{-5}$ early after surgery, but it rose again to $738 \pm 214$ dyne $\cdot \mathrm{s} \cdot \mathrm{cm}^{-5}$ at follow-up.

Figure 1 shows freedoms from all-cause death, diseasespecific death, and adverse events (including diseasespecific death and impaired functional status, NYHA functional class III). The values for freedom from disease-specific death at 5 and 10 years were $84 \%$ and $82 \%$, respectively, whereas those for freedom from adverse events were $78 \%$ and $70 \%$.

We next sought to determine risk factors for late adverse events. In the individual variable model, age and postoperative MPAP and PVR were associated with adverse events; however, only postoperative mPAP was found to be significant in the multivariable analysis (hazard ratio, 1.12; $95 \%$ confidence interval, $1.03-1.21 ; P<.01)$. Receiver operating characteristic curve analysis revealed a postoperative mPAP of $34 \mathrm{~mm} \mathrm{Hg}$ as the cutoff value for adverse events (area under the curve, 0.90; sensitivity, 0.80; specificity, $0.91)$.

Finally, the postoperative mPAP cutoff value was used to divide patients into 2 groups. There were no intergroup differences in any preoperative variables, although patients with a postoperative $\mathrm{mPAP}$ of at least $34 \mathrm{~mm} \mathrm{Hg}$ had a trend toward higher preoperative mPAP values than did those with a postoperative mPAP lower than $34 \mathrm{~mm} \mathrm{Hg} \mathrm{(50 \pm}$ $10 \mathrm{~mm} \mathrm{Hg}$ vs $45 \pm 10 \mathrm{~mm} \mathrm{Hg} ; P=.10$ ). Comparison of freedom rates from late adverse events between the groups (Figure 2) revealed that patients with postoperative mPAP values lower than $34 \mathrm{~mm} \mathrm{Hg}$ had good late outcomes. In that group, 10-year freedoms from disease-specific death and adverse events were $100 \%$ and $98 \%$, respectively. In contrast, patients with postoperative mPAP values of at least $34 \mathrm{~mm} \mathrm{Hg}$ had significant adverse events after PEA, and 10year freedoms from disease-specific death and adverse events were $80 \%$ and $41 \%$, respectively.

\section{DISCUSSION}

Medical treatment for CTEPH is palliative and unsatisfactory, but PEA is an effective therapeutic option that results in immediate and substantial improvements in pulmonary hemodynamics. This procedure is technically demanding, however, and requires proper patient selection and careful postoperative management, resulting in such relatively high in-hospital mortalities as $4.4 \%$ to $16 \%{ }^{1,4,6,10,14,15}$ Although our overall in-hospital mortality of $14 \%$ may be relatively high, the rate was reduced to $7.5 \%$ in the last 40 cases with increasing surgical experience and refinements in operative and postoperative management.

Several risk factors for increased in-hospital mortality have been identified, including advanced age, severe pulmonary hemodynamic compromise, CTEPH type 3 or 4 , distinct medical conditions involving other organ systems, and postoperative PVR greater than 500 dyne $\cdot \mathrm{s} \cdot \mathrm{cm}^{-5} \cdot 1,4,6,13,14,16$ In this study, only high PVR was identified as a preoperative risk factor, with a PVR cutoff point of 1052 dyne $\cdot \mathrm{s} \cdot \mathrm{cm}^{-5}$ for inhospital death. Although other work has shown that postoperative PVR greater than 500 dyne $\cdot \mathrm{s} \cdot \mathrm{cm}^{-5}$ may be a significant risk factor for in-hospital death, ${ }^{4}$ we did not evaluate postoperative PVR because there were 5 patients who could not be weaned from cardiopulmonary bypass.

TABLE 2. Risk factors for hospital mortality

\begin{tabular}{|c|c|c|c|c|}
\hline Variable & Survivors $(n=66)$ & Nonsurvivors $(\mathbf{n}=\mathbf{1 1})$ & $\begin{array}{c}\text { Univariate } \\
P \text { value }\end{array}$ & $\begin{array}{c}\text { Multivariate } \\
P \text { value }\end{array}$ \\
\hline Male (no.) & $27(41 \%)$ & $7(63 \%)$ & .20 & - \\
\hline Duration of illness (mo, mean $\pm \mathrm{SD}$ ) & $47 \pm 40$ & $62 \pm 39$ & .27 & - \\
\hline Mean pulmonary arterial pressure $(\mathrm{mm} \mathrm{Hg}$, mean $\pm \mathrm{SD}$ ) & $46 \pm 10$ & $54 \pm 9$ & .02 & - \\
\hline Pulmonary vascular resistance $\left(\right.$ dyne $\cdot \mathrm{s} \cdot \mathrm{cm}^{-5}$, mean $\pm \mathrm{SD}$ ) & $825 \pm 303$ & $1124 \pm 303$ & $<.01$ & $<.01$ \\
\hline Cardiac index $(\mathrm{L} /[\mathrm{min} \cdot \mathrm{m} 2]$, mean $\pm \mathrm{SD})$ & $2.5 \pm 0.7$ & $2.1 \pm 0.5$ & .33 & - \\
\hline \multicolumn{5}{|l|}{ Chronic thromboembolic pulmonary hypertension type (no.) } \\
\hline 1 & $52(87 \%)$ & $8(14 \%)$ & & \\
\hline 2 & $12(100 \%)$ & $0(0 \%)$ & & \\
\hline 3 & $2(50 \%)$ & $2(50 \%)$ & $.10 *$ & - \\
\hline
\end{tabular}

*Versus chronic thromboembolic pulmonary hypertension types 1 and 2. 

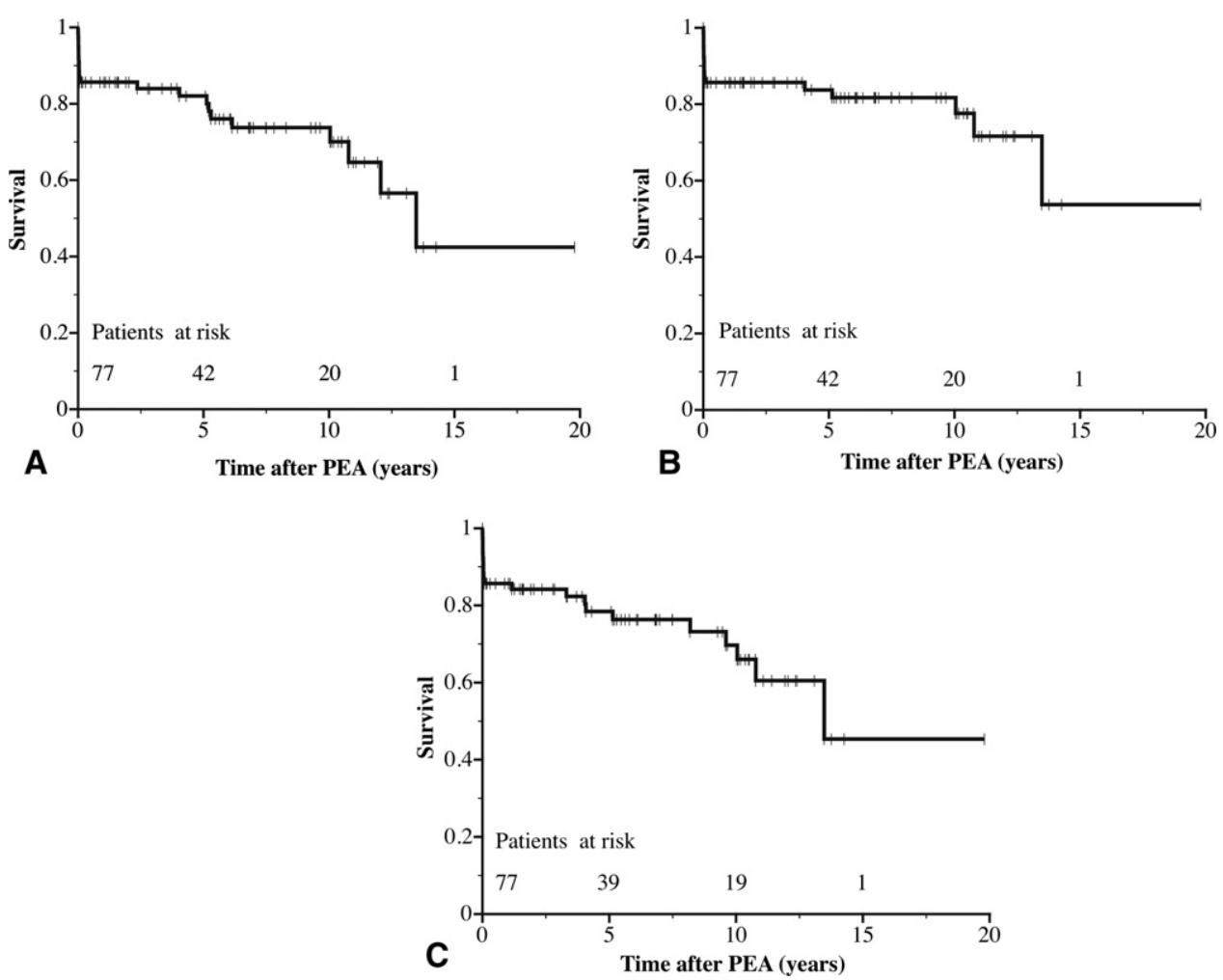

FIGURE 1. Overall Kaplan-Meier survival curves after pulmonary endarterectomy (PEA). A, Freedom from all-cause mortality. B, Freedom from diseasespecific mortality. C, Freedom from disease-specific mortality or New York Heart Association functional class III.

A previous study revealed that patients with a PVR greater than 1100 dyne $\cdot \mathrm{s} \cdot \mathrm{cm}^{-5}$ had 6 times higher operative mortality $(37 \%)$ than did those with a PVR lower than 1100 dyne $\cdot \mathrm{s} \cdot \mathrm{cm}^{-5} \cdot{ }^{16}$ Another study by Dartevelle and colleagues ${ }^{1}$ showed that the mortality was $4 \%$ among patients with PVR lower than 900 dyne $\cdot \mathrm{s} \cdot \mathrm{cm}^{-5}$; however, this increased to $10 \%$ among those with PVR between 900 and 1200 dyne $\cdot \mathrm{s} \cdot \mathrm{cm}^{-5}$ and to $20 \%$ among those with higher PVR. High PVR and CTEPH type 3 have been shown to be associated with persistent pulmonary hypertension, which is among the major complications after PEA and the leading cause of inhospital death. ${ }^{1,4}$ In a study by Thistlethwaite and associates, ${ }^{13}$ patients with CTEPH type 3 disease had higher rates of perioperative mortality and morbidity and smaller decreases in PAP and PVR than did those with proximal disease (type 1 or 2). Likewise, Freed and colleagues ${ }^{17}$ reported less improvement in pulmonary hemodynamics among patients with CTEPH type 3: 10 of their 17 patients with type 3 disease had postoperative persistent pulmonary hypertension, with mPAP values greater than $30 \mathrm{~mm} \mathrm{Hg}$. In the our study, it was not
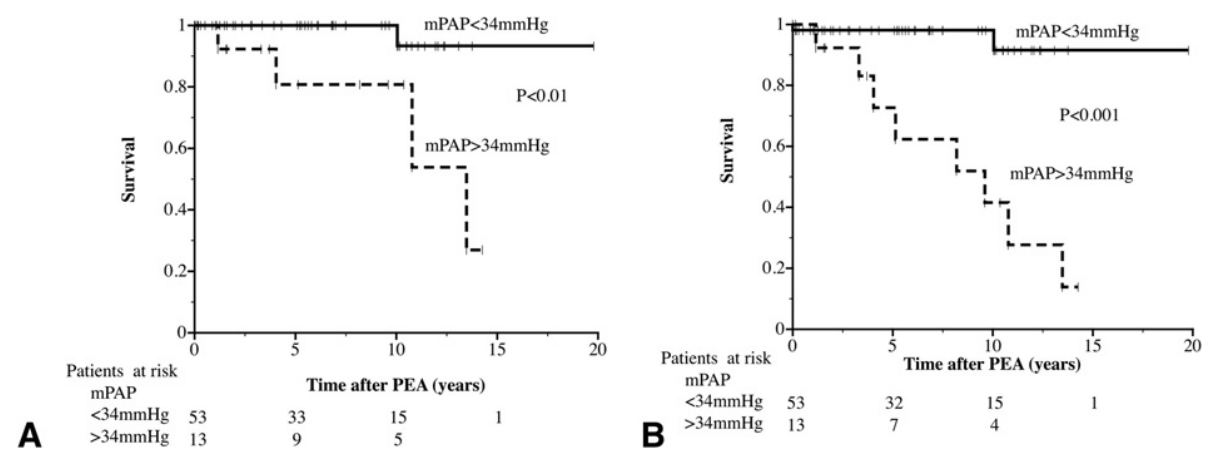

FIGURE 2. Kaplan-Meier survival curves after pulmonary endarterectomy ( $P E A)$ according to postoperative mean pulmonary arterial pressure ( $m P A P)$. A, Freedom from disease-specific mortality. B, Freedom from disease-specific mortality or New York Heart Association functional class III. 
conclusive whether CTEPH type 3 was associated with in-hospital mortality, because only a small number of patients had CTEPH type 3. Proper patient selection and careful perioperative management, however, are necessary when treating patients who have a high MPAP as a result of CTEPH type 3.

In addition to immediate improvements in pulmonary hemodynamics, PEA reportedly provides an excellent medium-term survival benefit, with 5-year survivals ranging from $75 \%$ to $89 \% .^{6-9}$ In a long-term follow-up of 308 patients who underwent PEA between 1970 and 1994, a University of California, San Diego, group found that $75 \%$ survived beyond 6 years. ${ }^{9}$ More recently, Corsico and coworkers ${ }^{8}$ reported the late results obtained for $157 \mathrm{pa}$ tients who underwent PEA between 1994 and 2006. They showed an $84 \%$ survival at 5 years, with a 30 -day mortality of $11.5 \%$. Our study, which reviewed the 20-year follow-up outcomes of patients who underwent PEA, has confirmed the persistent beneficial effect of PEA, as indicated by our late survival data. Indeed, 10-year survival was as high as $82 \%$ (Figure 1, $B$ ).

Patients with CTEPH are severely clinically compromised as a result of ventilation-perfusion mismatch and decreased cardiac output. In previous studies, more than $90 \%$ were in NYHA functional class III or IV, and most required oxygen therapy. ${ }^{6,8,14}$ Despite that clinically unpromising background, PEA provided significant and sustained improvements in clinical symptoms, with more than $90 \%$ of patients being in NYHA functional class I or II and 2 thirds having been weaned from oxygen therapy at the latest follow-up. ${ }^{6,8}$ In addition to prompt pulmonary hemodynamic improvement, PEA gradually improves gas exchange through a period of 6 months to 2 years after a temporal ventilation-perfusion abnormality caused by restrictive pulmonary functional impairment in response to surgical trauma, diffusion limitation in response to pulmonary edema, and a steal of perfusion from highresistance nonobstructed segment to low-resistance newly perfused segements. ${ }^{18,19}$ In our study, which was consistent with the previous results, only $63 \%$ of patients could be weaned from oxygen therapy, although $92 \%$ of patients were in NYHA functional class I or II. The discrepancy between excellent functional status and frequent oxygen therapy at follow-up can be explained by the fact that 10 of 21 patients with home oxygen therapy had a short follow-up period of less than 2 years.

Although a previous follow-up study found that persistent pulmonary hypertension and recurrent pulmonary embolism were the leading causes of late death, ${ }^{9}$ the risk factors for late adverse events after PEA remain to be identified because the data on long-term survival are relatively scarce. In one of the few relevant reports, Bonderman and associates ${ }^{15}$ showed that distinct medical conditions causing chronic infection or chronic inflammatory processes were risk factors for persistent pulmonary hypertension after PEA and were associated with both short- and long-term adverse outcomes. Freed and colleagues ${ }^{17}$ who reviewed medium-term follow-up results, showed that although persistent pulmonary hypertension with an mPAP value greater than $30 \mathrm{~mm} \mathrm{Hg}$ led to impairments in both functional status and exercise capacity, it had no adverse impact on 5-year survival. Our long-term follow-up study showed that persistence and worsening of pulmonary hypertension were associated with late death or impairment of functional status and identified postoperative mPAP as a risk factor for late adverse events, whereas the preoperative risk factors for inhospital death were not associated with late adverse events.

It has been shown that mPAP determines the prognosis of patients with medically treated CTEPH. ${ }^{3}$ Actually, in previous studies, mPAP values greater than $30 \mathrm{~mm} \mathrm{Hg}$ adversely affected survival in patients with medically treated CTEPH, whereas borderline pulmonary hypertension (20-26 mm $\mathrm{Hg}$ ) was not associated with a poor prognosis. ${ }^{3,20}$ In our study, a postoperative mPAP value of at least $34 \mathrm{~mm} \mathrm{Hg}$ was found to be the cutoff value for late adverse events. To judge from these results, a high mPAP determines the prognosis of patients with CTEPH, whether the disease is treated surgically or medically, and thus a decrease of mPAP is the most important goal if we hope to achieve good late survival. In contrast to the poor outcomes among patients with persistent pulmonary hypertension, an excellent 10-year event-free survival (98\%) was achieved among patients with resolved pulmonary hypertension (Figure 2). Our result indicates that PEA can be a curative and definitive surgical treatment and may be particularly important for patients with CTEPH, who are generally middle-aged (mean age around 52-57 years). ${ }^{1,4,6,10,14,15}$

Persistent pulmonary hypertension develops in $10 \%$ to $35 \%$ of patients who have undergone PEA, despite removal of sufficient proximal thromboembolic materials. ${ }^{6,10,14,17}$ Pathologic examination of lung tissue in patients with CTEPH has shown that small vessel arteriopathy occurs not only in the area served by open proximal arteries but also in the area distal to occluded pulmonary arteries. $^{1,21,22}$ This small vessel arteriopathy causes progressive pulmonary hypertension and a symptomatic decline in the course of the CTEPH and is related to the development of persistent pulmonary hypertension after a successful PEA. ${ }^{1,22}$ A reliable preoperative diagnostic tool for the involvement of distal arteriopathy has not been established ${ }^{2,20,22}$; however, patients who have a PVR that is disproportionately high with respect to the degree of proximal obstructions seen on pulmonary angiography are likely to have significant distal arteriopathy. ${ }^{1,2,4}$ These patients have an elevated risk of persistent pulmonary hypertension and therefore may be selected for PEA only if a $50 \%$ reduction in PVR is predicted. ${ }^{1}$ 
In conclusion, in our experience PEA provided immediate and substantial improvements in pulmonary hemodynamics and had sustained favorable effects on long-term survival. High PVR was a significant independent risk factor for in-hospital death. Persistence and worsening of pulmonary hypertension were associated with late death or impairment of functional class, and postoperative mPAP was shown as a risk factor for late adverse events, with an mPAP value of at least $34 \mathrm{~mm} \mathrm{Hg}$ being identified as the cutoff value for the prediction of such late adverse events.

\section{References}

1. Dartevelle P, Fadel E, Mussot S, Chapelier A, Hervé P, de Perrot M, et al. Chronic thromboembolic pulmonary hypertension. Eur Respir J. 2004;23:637-48.

2. Hoeper MM, Mayer E, Simonneau G, Rubin LJ. Chronic thromboembolic pulmonary hypertension. Circulation. 2006;113:2011-20.

3. Riedel M, Stanek V, Widimsky J, Prerovsky I. Longterm follow-up of patients with pulmonary thromboembolism. Late prognosis and evolution of hemodynamic and respiratory data. Chest. 1982;81:151-8.

4. Jamieson SW, Kapelanski DP, Sakakibara N, Manecke GR, Thistlethwaite PA, Kerr KM, et al. Pulmonary endarterectomy: experience and lessons learned in 1,500 cases. Ann Thorac Surg. 2003;76:1457-64.

5. Freed DH, Thomson BM, Tsui SS, Dunning JJ, Sheares KK, Pepke-Zaba J, et al. Functional and haemodynamic outcome 1 year after pulmonary thromboendarterectomy. Eur J Cardiothorac Surg. 2008;34:525-30.

6. Ogino H, Ando M, Matsuda H, Minatoya K, Sasaki H, Nakanishi N, et al. Japanese single-center experience of surgery for chronic thromboembolic pulmonary hypertension. Ann Thorac Surg. 2006;82:630-6.

7. Saouti N, Morshuis WJ, Heijmen RH, Snijder RJ. Long-term outcome after pulmonary endarterectomy for chronic thromboembolic pulmonary hypertension: a single institution experience. Eur J Cardiothorac Surg. 2009;35:947-52.

8. Corsico AG, D'Armini AM, Cerveri I, Klersy C, Ansaldo E, Niniano R, et al. Long-term outcome after pulmonary endarterectomy. Am J Respir Crit Care Med. 2008;15(178):419-24
9. Archibald CJ, Auger WR, Fedullo PF, Channick RN, Kerr KM, Jamieson SW, et al. Long-term outcome after pulmonary thromboendarterectomy. Am J Respir Crit Care Med. 1999;160:523-8.

10. Condliffe R, Kiely DG, Gibbs JS, Corris PA, Peacock AJ, Jenkins DP, et al. Improved outcomes in medically and surgically treated chronic thromboembolic pulmonary hypertension. Am J Respir Crit Care Med. 2008;177:1122-7.

11. Jamieson SW, Auger WR, Fedullo PF, Channick RN, Kriett JM, Tarazi RY, et al. Experience and results with 150 pulmonary thromboendarterectomy operations over a 29-month period. J Thorac Cardiovasc Surg. 1993;106:116-27.

12. Jamieson SW, Kapelanski DP. Pulmonary endarterectomy. Curr Probl Surg. 2000;37:165-252.

13. Thistlethwaite PA, Mo M, Madani MM, Deutsch R, Blanchard D, Kapelanski DP, et al. Operative classification of thromboembolic disease determines outcome after pulmonary endarterectomy. J Thorac Cardiovasc Surg. 2002;124(6):1203-11.

14. Tscholl D, Langer F, Wendler O, Wilkens H, Georg T, Schäfers HJ. Pulmonary thromboendarterectomy-risk factors for early survival and hemodynamic improvement. Eur J Cardiothorac Surg. 2001;19:771-6.

15. Bonderman D, Skoro-Sajer N, Jakowitsch J, Adlbrecht C, Dunkler D, Taghavi S, et al. Predictors of outcome in chronic thromboembolic pulmonary hypertension. Circulation. 2007; 115:2153-8

16. Hartz RS, Byrne JG, Levitsky S, Park J, Rich S. Predictors of mortality in pulmonary thromboendarterectomy. Ann Thorac Surg. 1996;62:1255-60.

17. Freed DH, Thomson BM, Berman M, Tsui SS, Dunning J, Sheares KK, et al. Survival after pulmonary thromboendarterectomy: Effect of residual pulmonary hypertension. J Thorac Cardiovasc Surg. 2011;141:383-7.

18. Kapitan KS, Clausen JL, Moser KM. Gas exchange in chronic thromboembolism after pulmonary thromboendarterectomy. Chest. 1990;98:14-9.

19. Tanabe N, Okada O, Nakagawa Y, Masuda M, Kato K, Nakajima N, et al. The efficacy of pulmonary thromboendarterectomy on long-term gas exchange. Eur Respir J. 1997;10:2066-72.

20. Lewczuk J, Piszko P, Jagas J, Porada A, Wójciak S, Sobkowicz B, et al. Prognostic factors in medically treated patients with chronic pulmonary embolism. Chest. 2001;119:818-23.

21. Moser KM, Bloor CM. Pulmonary vascular lesions occurring in patients with chronic major vessel thromboembolic pulmonary hypertension. Chest. 1993; 103:685-92.

22. Galiè N, Kim NH. Pulmonary microvascular disease in chronic thromboembolic pulmonary hypertension. Proc Am Thorac Soc. 2006;3:571-6. 